\title{
Collocation Method Based on Genocchi Operational Matrix for Solving Generalized Fractional Pantograph Equations
}

\author{
Abdulnasir Isah, ${ }^{1,2}$ Chang Phang, ${ }^{2}$ and Piau Phang ${ }^{3}$ \\ ${ }^{1}$ Department of Mathematics, Ahmadu Bello University, Zaria, Nigeria \\ ${ }^{2}$ Department of Mathematics and Statistics, Faculty of Science, Technology and Human Development, \\ Universiti Tun Hussein Onn Malaysia, Batu Pahat, Malaysia \\ ${ }^{3}$ Faculty of Computer Science and Information Technology, Universiti Malaysia Sarawak, Sarawak, Malaysia
}

Correspondence should be addressed to Piau Phang; pphang@unimas.my

Received 2 January 2017; Revised 16 March 2017; Accepted 16 May 2017; Published 13 June 2017

Academic Editor: Patricia J. Y. Wong

Copyright (C) 2017 Abdulnasir Isah et al. This is an open access article distributed under the Creative Commons Attribution License, which permits unrestricted use, distribution, and reproduction in any medium, provided the original work is properly cited.

An effective collocation method based on Genocchi operational matrix for solving generalized fractional pantograph equations with initial and boundary conditions is presented. Using the properties of Genocchi polynomials, we derive a new Genocchi delay operational matrix which we used together with the Genocchi operational matrix of fractional derivative to approach the problems. The error upper bound for the Genocchi operational matrix of fractional derivative is also shown. Collocation method based on these operational matrices is applied to reduce the generalized fractional pantograph equations to a system of algebraic equations. The comparison of the numerical results with some existing methods shows that the present method is an excellent mathematical tool for finding the numerical solutions of generalized fractional pantograph equations.

\section{Introduction}

Fractional calculus, the calculus of derivative and integral of any order, is used as a powerful tool in science and engineering to study the behaviors of real world phenomena especially the ones that cannot be fully described by the classical methods and techniques [1]. Differential equations with proportional delays are usually referred to as pantograph equations or generalized pantograph equations. The name pantograph was originated from the study work of Ockendon and Tayler [2]. Many researchers have studied different applications of these equations in applied sciences such as biology, physics, economics, and electrodynamics [3-5]. Solutions of pantograph equations were also studied by many authors numerically and analytically. Bhrawy et al. proposed a new generalized Laguerre-Gauss collocation method for numerical solution of generalized fractional pantograph equations [1]. Tohidi et al. in [6] proposed a new collocation scheme based on Bernoulli operational matrix for numerical solution of generalized pantograph equation. Yusufoglu [7] proposed an efficient algorithm for solving generalized pantograph equations with linear functional argument. In [8], Yang and Huang presented a spectral-collocation method for fractional pantograph delay integrodifferential equations and in [9] Yüzbasi and Sezer presented an exponential approximation for solutions of generalized pantograph delay differential equations. Chebyshev and Bessel polynomials are, respectively, used in $[10,11]$ to obtain the solutions of generalized pantograph equations. Operational matrices of fractional derivatives and integration have become very important tool in the field of numerical solution of fractional differential equations. In this paper, a member of Appell polynomials called Genocchi polynomials is used; although this polynomial is not based on orthogonal functions, it possesses operational matrices of derivatives with high accuracy. It is very important to note that this polynomial shares some great advantages with Bernoulli and Euler polynomials for approximating an arbitrary function over some classical orthogonal polynomials; we refer the reader to [6] for these advantages. On top of that, we 
had successfully applied the operational matrix via Genocchi polynomials for solving integer-order delay differential equations [12] and fractional optimal control problems [13], and the numerical solutions obtained are comparable or even more accurate compared to some existing well-known methods. Motivated by these advantages, in this paper, we intend to extend the result for integer-order delay differential equations in [12] to fractional delay differential equations or so-called generalized fractional pantograph equations. To the best of our knowledge, this is the first time that the operational matrix based on Genocchi polynomials is applied to solve the fractional pantograph equations. On the other hand, some other types of polynomials were employed to solve some special type of fractional calculus problems; for example, Bessel polynomials were used for the solution of fractional-order logistic population model [14]; Bernstein polynomials were also used for the solution of Riccati type differential equations [15].

In this paper, we use the new operational matrix of fractional-order derivative via Genocchi polynomials to provide approximate solutions of the generalized fractional pantograph equations of the following form [1]:

$$
\begin{aligned}
D^{\alpha} y(t)=\sum_{j=0}^{J} \sum_{n=0}^{m-1} p_{j, n}(t) D^{\beta_{n}} y\left(\lambda_{j, n} t+\mu_{j, n}\right)+ & g(t), \\
& 0 \leq t \leq 1
\end{aligned}
$$

subject to the following conditions:

$$
\sum_{n=0}^{m-1} a_{n, i} y^{(n)}(0)=d_{i}, \quad i=0,1, \ldots, m-1,
$$

where $a_{n, i}, \quad \lambda_{j, n}$, and $\mu_{j, n}$ are real or complex coefficients; $m-$ $1<\alpha<m, 0<\beta_{0}<\beta_{1}<\cdots<\beta_{m-1}<\alpha$, while $p_{j, n}(t)$ and $g(t)$ are given continuous functions in the interval $[0,1]$.

The rest of the paper is organized as follows: Section 2 introduces some mathematical preliminaries of fractional calculus. In Section 3, we discuss some important properties of Genocchi polynomials. In Section 4, we derive the Genocchi delay operational matrix and we apply the collocation method for solving fractional pantograph equation (1) using the Genocchi operational matrix of fractional derivative and the delay operational matrix in Section 5. In Section 6, the proposed method is applied to several examples and conclusion is given in Section 7.

\section{Preliminaries}

2.1. Fractional Derivative and Integration. We recall some basic definitions and properties of fractional calculus that we will use. There are various competing definitions for fractional derivatives $[16,17]$. The Riemann-Liouville definition played a vital role in the development of the theory of fractional calculus. However, there are certain disadvantages of using this definition when modeling real world phenomena. To cope with these disadvantages, Caputo definition was introduced which is found to be more reliable in application. So we use this definition of fractional derivatives. We begin with the definition of Riemann-Liouville integral, in which the fractional integral operator $I$ of a function $f(t)$ is defined as follows.

Definition 1. The Riemann-Liouville integral $I$ of fractionalorder $\alpha$ of $f(t)$ is given by

$$
I^{\alpha} f(t)=\frac{1}{\Gamma(\alpha)} \int_{0}^{t}(t-\tau)^{\alpha-1} f(\tau) d \tau,
$$

$$
t>0, \alpha \in \mathbb{R}^{+},
$$

where $\Gamma(\cdot)$ is the Gamma function. The fractional derivative of order $\alpha>0$ due to Riemann-Liouville is defined by

$$
\left(D_{l}^{\alpha} f\right)(t)=\left(\frac{d}{d t}\right)^{m}\left(I^{m-\alpha} f\right)(t),
$$

$$
(\alpha>0, m-1<\alpha<m) \text {. }
$$

The following are important properties of Riemann-Liouville fractional integral $I^{\alpha}$ :

$$
\begin{aligned}
I^{\alpha} I^{\beta} f(t) & =I^{\alpha+\beta} f(t), \quad \alpha>0, \beta>0, \\
I^{\alpha} t^{\beta} & =\frac{\Gamma(\beta+1)}{\Gamma(\beta+\alpha+1)} t^{\beta+\alpha} .
\end{aligned}
$$

Definition 2. The Caputo fractional derivative $D^{\alpha}$ of a function $f(t)$ is defined as

$$
\begin{array}{r}
D^{\alpha} f(t)=\frac{1}{\Gamma(n-\alpha)} \int_{0}^{t} \frac{f^{(n)}(\tau)}{(t-\tau)^{\alpha-n+1}} d \tau, \\
n-1<\alpha \leq n, n \in \mathbb{N} .
\end{array}
$$

Some properties of Caputo fractional derivatives are as follows:

$$
\begin{aligned}
& D^{\alpha} C=0, \quad(C \text { is constant }), \\
& D^{\alpha} t^{\beta}= \begin{cases}0, & \beta \in \mathbb{N} \cup\{0\}, \beta<\lceil\alpha\rceil \\
\frac{\Gamma(\beta+1)}{\Gamma(\beta+1-\alpha)} t^{\beta-\alpha}, & \beta \in \mathbb{N} \cup\{0\}, \beta \geq\lceil\alpha\rceil \text { or } \beta \notin \mathbb{N}, \beta>\lfloor\alpha\rfloor,\end{cases}
\end{aligned}
$$


where $\lceil\alpha\rceil$ denotes the smallest integer greater than or equal to $\alpha$ and $\lfloor\alpha\rfloor$ denotes the largest integer less than or equal to $\alpha$.

Similar to the integer-order differentiation, the Caputo fractional differential operator is a linear operator; that is,

$$
D^{\alpha}(\lambda f(t)+\mu g(t))=\lambda D^{\alpha} f(t)+\mu D^{\alpha} g(t)
$$

for $\lambda$ and $\mu$ constants.

\section{Genocchi Polynomials and Some Properties}

Genocchi polynomials and numbers have been extensively studied in many different contexts in branches of mathematics such as elementary number theory, complex analytic number theory, homotopy theory (stable homotopy groups of spheres), differential topology (differential structures on spheres), theory of modular forms (Eisenstein series), and quantum physics (quantum groups). The classical Genocchi polynomial $G_{n}(x)$ is usually defined by means of the exponential generating functions [18-20].

$$
\frac{2 t e^{x t}}{e^{t}+1}=\sum_{n=0}^{\infty} G_{n}(x) \frac{t^{n}}{n !}, \quad(|t|<\pi)
$$

where $G_{n}(x)$ is the Genocchi polynomial of degree $n$ and is given by

$$
G_{n}(x)=\sum_{k=0}^{n}\left(\begin{array}{l}
n \\
k
\end{array}\right) G_{n-k} x^{k}
$$

$G_{n-k}$ here is the Genocchi number.

Some of the important properties of these polynomials include

$$
\begin{aligned}
\int_{0}^{1} G_{n}(x) G_{m}(x) d x & =\frac{2(-1)^{n} n ! m !}{(m+n) !} G_{m+n} \quad n, m \geq 1, \\
\frac{d G_{n}(x)}{d x} & =n G_{n-1}(x), \quad n \geq 1, \\
G_{n}(1)+G_{n}(0) & =0, \quad n>1 .
\end{aligned}
$$

Before we move to the next level, we need the following linear independence on which the rest of theoretical results are based.

Lemma 3. The set $A=\left\{G_{1}(t), G_{2}(t), \ldots, G_{N}(t)\right\} \subset L^{2}[0,1]$ is a linearly independent set in $L^{2}[0,1]$.

Proof. To show that $A$ is the set of linearly independent elements of $L^{2}[0,1]$, it is enough to show that the Gram determinant is not zero. That is,

$$
\operatorname{Gram}\left(G_{1}, G_{2}, \ldots, G_{N}\right) \neq 0,
$$

where

$$
\begin{aligned}
& \operatorname{Gram}\left(G_{1}, G_{2}, \ldots, G_{N}\right) \\
& =\left|\begin{array}{cccc}
\left\langle G_{1}, G_{1}\right\rangle & \left\langle G_{1}, G_{2}\right\rangle & \ldots & \left\langle G_{1}, G_{N}\right\rangle \\
\left\langle G_{2}, G_{1}\right\rangle & \left\langle G_{2}, G_{2}\right\rangle & \ldots & \left\langle G_{2}, G_{N}\right\rangle \\
\vdots & \vdots & \ldots & \vdots \\
\left\langle G_{n}, G_{1}\right\rangle & \left\langle G_{n}, G_{2}\right\rangle & \ldots & \left\langle G_{n}, G_{N}\right\rangle
\end{array}\right| .
\end{aligned}
$$

Now, to prove that this determinant is not equal to zero, we first reduce the Gram matrix to an upper triangular matrix by Gaussian elimination and it is not difficult to see that the elements of the diagonal of the reduced matrix are given by

$$
a(n)=\frac{(n !(n+1) !)^{2}}{(2 n !)(2 n+1) !}, \quad n \in \mathbb{N} .
$$

Clearly, one can see that, for any $\in \mathbb{N}, a(n) \neq 0$. Consequently, the determinant given by

$$
\prod_{n=1}^{N} a(n)
$$

is not equal to zero. Therefore, the set $A$ is the set of linearly independent sets.

3.1. Function Approximation. Assume that $\left\{G_{1}(t), G_{2}(t)\right.$, $\left.\ldots, G_{N}(t)\right\} \subset L^{2}[0,1]$ is the set of Genocchi polynomials and $Y=\operatorname{Span}\left\{G_{1}(t), G_{2}(t), \ldots, G_{N}(t)\right\}$. Let $f(t)$ be arbitrary element of $L^{2}[0,1]$; since $Y$ is a finite dimensional subspace of $L^{2}[0,1]$ space, $f(t)$ has a unique best approximation in $Y$, say $f^{*}(t)$, such that

$$
\left\|f(t)-f^{*}(t)\right\|_{2} \leq\|f(t)-y(t)\|_{2}, \quad \forall y(t) \in Y .
$$

This implies that, $\forall y(t) \in Y$,

$$
\left\langle f(t)-f^{*}(t), y(t)\right\rangle=0,
$$

where $\langle\cdot\rangle$ denotes inner product. Since $f^{*}(t) \in Y$, there exist the unique coefficients $c_{1}, c_{2}, \ldots, c_{N}$ such that

$$
f(t) \approx f^{*}(t)=\sum_{n=1}^{N} c_{n} G_{n}(t)=\mathbf{C}^{T} \mathbf{G}(t),
$$

where $\mathbf{C}=\left[c_{1}, c_{2}, \ldots, c_{N}\right]^{T}, \mathbf{G}(t)=\left[G_{1}(t), G_{2}(t), \ldots\right.$, $\left.G_{N}(t)\right]^{T}$.

Using (19), we have

$$
\left\langle f(t)-\mathbf{C}^{T} \mathbf{G}(t), G_{i}(t)\right\rangle=0 \quad i=1,2, \ldots, N ;
$$

for simplicity, we write

$$
\mathbf{C}^{T}\langle\mathbf{G}(t), \mathbf{G}(t)\rangle=\langle f(t), \mathbf{G}(t)\rangle,
$$

where $\langle\mathbf{G}(t), \mathbf{G}(t)\rangle$ is an $N \times N$ matrix.

$$
\text { Let } W=\langle\mathbf{G}(t), \mathbf{G}(t)\rangle=\int_{0}^{1} \mathbf{G}(t) \mathbf{G}^{T}(t) d t .
$$

The entries of the matrix $W$ can be calculated from (11). Therefore, any function $f(t) \in L^{2}[0,1]$ can be expanded by Genocchi polynomials as $f(t)=\mathbf{C}^{T} \mathbf{G}(t)$, where

$$
\mathbf{C}=W^{-1}\langle f(t), \mathbf{G}(t)\rangle \text {. }
$$




\section{Genocchi Operational Matrix}

In this section, we derive the operational matrices for the delay and that of fractional derivative based on Genocchi polynomials for the solution of fractional pantograph equations.

4.1. Genocchi Delay Operational Matrix. The Genocchi delay vector $\mathbf{G}(t-\mu)$ can be expressed as

$$
\mathbf{G}(t-\mu)=\mathbf{R} \mathbf{G}(t)
$$

where $\mathbf{R}$ is the $N \times N$ operational delay matrix given by

$$
\begin{aligned}
\mathbf{R} & =W_{1} W^{-1} \\
& =\left[\begin{array}{ccccccc}
1 & 0 & 0 & 0 & 0 & \cdots & 0 \\
-2 \mu & 1 & 0 & 0 & 0 & \cdots & 0 \\
3 \mu^{2} & -3 \mu & 1 & 0 & 0 & \cdots & 0 \\
-4 \mu^{3} & 6 \mu^{2} & -4 \mu & 1 & 0 & \cdots & 0 \\
5 \mu^{4} & -10 \mu^{3} & 10 \mu^{2} & -5 \mu & 1 & \cdots & 0 \\
\vdots & \vdots & \vdots & \ldots & \vdots & \vdots & \vdots \\
b_{n}(1) & b_{n}(2) & b_{n}(3) & b_{n}(4) & b_{n}(5) & \cdots & 1
\end{array}\right],
\end{aligned}
$$

where $W_{1}=\int_{0}^{1} \mathbf{G}(t-\mu) \mathbf{G}^{T}(t) d t$ and $b_{n}(i)=(-1)^{n-i}\left(\left(\begin{array}{c}n \\ i\end{array}\right)\right) \mu^{n-i}$, $i=1,2, \ldots, n$.

Also, for any delay function $f(t-\mu)$, we can express it in terms of Genocchi polynomials as shown in (26):

$$
f(t-\mu)=\sum_{i=1}^{N} c_{i} G_{i}(t-\mu)=\mathbf{C}^{T} \mathbf{R G}(t)
$$

where $\mathbf{C}$ is given in (23).

The following lemma is also of great importance.

Lemma 4. Let $G_{i}(t)$ be the Genocchi polynomials; then $D^{\alpha} G_{i}(t)=0$, for $i=1, \ldots,\lceil\alpha\rceil-1, \alpha>0$.

The proof of this lemma is obvious; one can use (7) and (8) on (10).

4.2. Genocchi Operational Matrix of Fractional Derivative. If we consider the Genocchi vector $\mathbf{G}(t)$ given by $\mathbf{G}(t)=$ $\left[G_{1}(t), G_{2}(t), \ldots, G_{N}(t)\right]$, then the derivative of $\mathbf{G}(t)$ with the aid of (12) can be expressed in the matrix form by

$$
\frac{d \mathbf{G}(t)^{T}}{d t}=M \mathbf{G}(t)^{T},
$$

where

$$
M=\left[\begin{array}{ccccccc}
0 & 0 & 0 & \cdots & 0 & 0 & 0 \\
2 & 0 & 0 & \cdots & 0 & 0 & 0 \\
0 & 3 & 0 & \cdots & 0 & 0 & 0 \\
0 & 0 & 4 & \cdots & 0 & 0 & 0 \\
\vdots & \vdots & \vdots & \cdots & \vdots & \vdots & \vdots \\
0 & 0 & 0 & \cdots & N-1 & 0 & 0 \\
0 & 0 & 0 & \cdots & 0 & N & 0
\end{array}\right] .
$$

Thus, $M$ is $N \times N$ operational matrix of derivative.

It is not difficult to show inductively that the $k$ th derivative of $\mathbf{G}(t)$ can be given by

$$
\frac{d^{k} \mathbf{G}(t)^{T}}{d t^{k}}=\mathbf{G}(t)\left(M^{T}\right)^{k}
$$

In the following theorem, the operational matrix of fractional-order derivative for the Genocchi polynomials is given.

Theorem 5 (see [21]). Suppose that $\mathbf{G}(t)$ is the Genocchi vector given in (20) and let $\alpha>0$. Then,

$$
D^{\alpha} \mathbf{G}(t)^{T}=P^{\alpha} \mathbf{G}(t)^{T}
$$

where $P^{\alpha}$ is $N \times N$ operational matrix of fractional derivative of order $\alpha$ in Caputo sense and is defined as follows:

$$
P^{(\alpha)}=\left[\begin{array}{cccc}
0 & 0 & \cdots & 0 \\
\vdots & \vdots & \cdots & \vdots \\
0 & 0 & \cdots & 0 \\
\sum_{k=\lceil\alpha\rceil}^{\lceil\alpha\rceil} \rho_{\lceil\alpha\rceil, k, 1} & \sum_{k=\lceil\alpha\rceil}^{\lceil\alpha\rceil} \rho_{\lceil\alpha\rceil, k, 2} & \cdots & \sum_{k=\lceil\alpha\rceil} \rho_{\lceil\alpha\rceil, k, N} \\
\vdots & \vdots & \cdots & \vdots \\
\sum_{k=\lceil\alpha\rceil}^{i} \rho_{i, k, 1} & \sum_{k=\lceil\alpha\rceil}^{i} \rho_{i, k, 2} & \cdots & \sum_{k=\lceil\alpha\rceil}^{i} \rho_{i, k, N} \\
\vdots & \vdots & \cdots & \vdots \\
\sum_{k=\lceil\alpha\rceil}^{N} \rho_{N, k, 1} & \sum_{k=\lceil\alpha\rceil}^{N} \rho_{N, k, 2} & \cdots & \sum_{k=\lceil\alpha\rceil}^{N} \rho_{N, k, N}
\end{array}\right],
$$

where $\rho_{i, k, j}$ is given by

$$
\rho_{i, k, j}=\frac{i ! G_{i-k}}{(i-k) ! \Gamma(k+1-\alpha)} c_{j} .
$$
(23)

$G_{i-k}$ is the Genocchi number and $c_{j}$ can be obtained from

Proof. For the proof, see [21]. 
4.3. Upper Bound of the Error for the Operational Matrix of Fractional Derivative $P^{\alpha}$. We begin here by proving the upper bound of the error of arbitrary function approximation by Genocchi polynomials in the following Lemma.

Lemma 6. Suppose that $f(t) \in C^{n+1}[0,1]$ and $Y=$ $\operatorname{Span}\left\{G_{1}(t), G_{2}(t), \ldots, G_{N}(t)\right\}$; if $\mathbf{C}^{T} \mathbf{G}(t)$ is the best approximation of $f(t)$ out of $Y$, then

$$
\left\|f(t)-\mathbf{C}^{T} \mathbf{G}(t)\right\| \leq \frac{R}{(n+1) ! \sqrt{2 n+3}},
$$

where $R=\max _{t \in[0,1]}\left|f^{(n+1)}(t)\right|$.

To see this, we set $\left\{1, t, \ldots, t^{n}\right\}$ as a basis for the polynomial space of degree $n$.

Define $y_{1}(t)=f(0)+t f^{\prime}(0)+\left(t^{2} / 2 !\right) f^{\prime \prime}(0)+\cdots+$ $\left(t^{n} / n !\right) f^{(n)}(0)$.

From Taylor's expansion, one has $\left|f(t)-y_{1}(t)\right|=\mid\left(t^{n+1} /(n+\right.$ 1)!) $f^{(n+1)}\left(\xi_{t}\right) \mid$, where $\xi_{t} \in(0,1)$.

Since $\mathbf{C}^{T} \mathbf{G}(t)$ is the best approximation of $f(t)$ out of $Y$ and $y_{1}(t) \in Y$, from (18), one has

$$
\begin{aligned}
& \left\|f(t)-\mathbf{C}^{T} \mathbf{G}(t)\right\|_{2}^{2} \leq\left\|f(t)-y_{1}(t)\right\|_{2}^{2} \\
& \quad \leq \int_{0}^{1}\left|f(t)-y_{1}(t)\right|^{2} d t \\
& \quad=\int_{0}^{1}\left(\frac{t^{n+1}}{(n+1) !}\right)^{2}\left\|f^{(n+1)}\left(\xi_{t}\right)\right\|^{2} d t \\
& \quad \leq \frac{R^{2}}{((n+1) !)^{2}} \int_{0}^{1} t^{2 n+2} d t=\frac{R^{2}}{((n+1) !)^{2}(2 n+3)} .
\end{aligned}
$$

Taking the square root of both sides, one has

$$
\left\|f(t)-\mathbf{C}^{T} \mathbf{G}(t)\right\| \leq \frac{R}{(n+1) ! \sqrt{2 n+3}}
$$

which is the desired error bound.

We use the following theorem from [22].

Theorem 7 (see [22]). Suppose that $H$ is a Hilbert space and $Y$ is a closed subspace of $H$ such that $\operatorname{dim} Y<\infty$ and $y_{1}, y_{2}, \ldots, y_{n}$ is a basis for $Y$. Let $f$ be an arbitrary element in $H$ and let $y_{0}$ be the unique best approximation of $f$ out of $Y$. Then,

$$
\left\|f-y_{0}\right\|^{2}=\frac{\operatorname{Gram}\left(f, y_{1}, y_{2}, \ldots, y_{n}\right)}{\operatorname{Gram}\left(y_{1}, y_{2}, \ldots, y_{n}\right)}
$$

where

$$
\begin{aligned}
& \operatorname{Gram}\left(y_{1}, y_{2}, \ldots, y_{n}\right) \\
& =\left|\begin{array}{cccc}
\left\langle y_{1}, y_{1}\right\rangle & \left\langle y_{1}, y_{2}\right\rangle & \ldots & \left\langle y_{1}, y_{n}\right\rangle \\
\left\langle y_{2}, y_{1}\right\rangle & \left\langle y_{2}, y_{2}\right\rangle & \ldots & \left\langle y_{2}, y_{n}\right\rangle \\
\vdots & \vdots & \ldots & \vdots \\
\left\langle y_{n}, y_{1}\right\rangle & \left\langle y_{n}, y_{2}\right\rangle & \ldots & \left\langle y_{n}, y_{n}\right\rangle
\end{array}\right| .
\end{aligned}
$$

Theorem 8. Suppose that $f(t) \in L^{2}[0,1]$ is approximated by $f_{n}(t)$ as

$$
f_{n}(t)=\sum_{i=1}^{n} c_{i} G_{i}(t)=\mathbf{C}^{T} \mathbf{G}(t)
$$

then,

$$
\lim _{n \rightarrow \infty}\left\|f(t)-f_{n}(t)\right\|=0
$$

The proof of this theorem obviously follows from Lemma 6.

The operational matrix error vector $E^{\alpha}$ is given by

$$
E^{\alpha}=P^{\alpha} \mathbf{G}(t)-D^{\alpha} \mathbf{G}(t),
$$

where

$$
E^{\alpha}=\left[\begin{array}{c}
e_{1}^{\alpha} \\
e_{2}^{\alpha} \\
\vdots \\
e_{n}^{\alpha}
\end{array}\right]
$$

from Theorem 7, we get

$$
\begin{gathered}
\left\|f_{0}(t)-\sum_{j=1}^{N} c_{j} G_{j}(t)\right\| \\
=\left(\frac{\operatorname{Gram}\left(f(t), G_{1}(t), \ldots, G_{N}(t)\right)}{\operatorname{Gram}\left(G_{1}(t), \ldots, G_{N}(t)\right)}\right)^{1 / 2} .
\end{gathered}
$$

Thus, according to equations (29) and (30) in [21], one has

$$
\begin{aligned}
\left\|e_{i}^{\alpha}\right\| & =\mid D^{\alpha} G_{i}(t) \\
& -\sum_{j=1}^{N}\left(\sum_{k=\lceil\alpha\rceil}^{i} \frac{i ! G_{i-k}}{(i-k) ! \Gamma(k+1-\alpha)} c_{j}\right) G_{j}(t) \mid \\
& \leq \sum_{k=\lceil\alpha\rceil}^{i} \frac{i ! G_{i-k}}{(i-k) ! \Gamma(k+1-\alpha)} t^{k-\alpha} \mid f_{0}(t) \\
& -\sum_{j=1}^{N} c_{j} G_{j}(t) \mid \leq \sum_{k=\lceil\alpha\rceil}^{i} \frac{i ! G_{i-k}}{(i-k) ! \Gamma(k+1-\alpha)} t^{k-\alpha} \\
& \cdot\left(\frac{\operatorname{Gram}\left(f(t), G_{1}(t), \ldots, G_{N}(t)\right)}{\operatorname{Gram}\left(G_{1}(t), \ldots, G_{N}(t)\right)}\right)^{1 / 2} .
\end{aligned}
$$

By considering Theorem 8 and (43), we can conclude that by increasing the number of the Genocchi bases the vector $e_{i}^{\alpha}$ tends to zero.

For comparison purpose in Table 1, we show below the errors of operational matrix of fractional derivative based on Genocchi polynomials and shifted Legendre polynomials derived in $[23,24]$ when $N=10$ and $\alpha=0.75$ at different 
TABLE 1: Comparison of the operational matrix errors for the GPOMFD and SLPOMFD.

\begin{tabular}{ccccccc}
\hline$E^{\alpha}$ & & $x=1$ & & & & \multicolumn{2}{c}{$x=0.5$} \\
& GPOMFD & SLPOMFD & GPOMFD & SLPOMFD & GPOMFD & SLPOMFD \\
\hline$e_{1}$ & 0.00000 & 0.00000 & 0.00000 & 0.00000 & 0.00000 & 0.00000 \\
$e_{2}$ & 0.01288 & 0.01343 & 0.51657 & 0.51648 & 0.00353 & 0.00345 \\
$e_{3}$ & 0.02204 & 0.03462 & 0.77767 & 1.56518 & 0.00531 & 0.00008 \\
$e_{4}$ & 0.00004 & 0.02916 & 0.01241 & 3.17608 & 0.00911 & 0.01047 \\
$e_{5}$ & 0.03944 & 0.17666 & 1.28048 & 5.36557 & 0.00001 & 0.025787 \\
$e_{6}$ & 0.00292 & 0.33223 & 0.02450 & 15.06874 & 0.05647 \\
$e_{7}$ & 0.15747 & 2.61748 & 5.38767 & 11.80719 & 0.00060 & 0.04226 \\
$e_{8}$ & 0.00748 & 11.8527 & 0.15766 & 54.74287 & 0.27897 & 0.00519 \\
$e_{9}$ & 1.21090 & 38.04476 & 39.19581 & 629.08480 & 15.64398 \\
$e_{10}$ & 0.04902 & 806.14232 & 1.32181 & 20.30772 \\
\hline
\end{tabular}

points on $[0,1]$. From this table, it is clear that the accuracy of Genocchi polynomials operational matrix of fractional derivative (GPOMFD) is better than the shifted Legendre polynomials operational matrix of fractional derivatives (SLPOMFD). We believe that this is the case for any value of $N$ because the Genocchi polynomials have smaller coefficients of individual terms compared to shifted Legendre polynomials.

\section{Collocation Method Based on Genocchi Operational Matrices}

In this section, we use the collocation method based on Genocchi operational matrix of fractional derivatives and Genocchi delay operational matrix to solve numerically the generalized fractional pantograph equation. We now derive an algorithm for solving (1). To do this, let the solution of (1) be approximated by the first $N$ terms Genocchi polynomials. Thus, we write

$$
y_{N}(t) \approx \sum_{n=1}^{N} c_{n} G_{n}(t)=G(t) C,
$$

where the Genocchi coefficient vector $C$ and the Genocchi vector $G(t)$ are given by

$$
\begin{aligned}
C^{T} & =\left[c_{1}, c_{2}, \ldots, c_{N}\right], \\
G(t) & =\left[G_{1}(t), G_{2}(t), \ldots, G_{N}(t)\right] ;
\end{aligned}
$$

thus, $D^{\alpha} y_{N}(t)$ and $D^{\beta_{n}} y_{N}(t), n=0,1, \ldots, m-1$, can be expressed, respectively, as follows:

$$
\begin{aligned}
D^{\alpha} y_{N}(t) & =G(t)\left(P^{T}\right)^{\alpha} C, \\
D^{\beta_{n}} y_{N}(t) & =G(t)\left(P^{T}\right)^{\beta_{n}} C, \quad n=0,1, \ldots, m-1 .
\end{aligned}
$$

Substituting (44) and (46) in (1), we have

$$
\begin{aligned}
G(t) & \left(P^{T}\right)^{\alpha} C \\
= & \sum_{j=0}^{J} \sum_{n=0}^{m-1} p_{j, n}(t) G\left(\lambda_{j, n} t+\mu_{j, n}\right)\left(P^{T}\right)^{\beta_{n}} C+g(t),
\end{aligned}
$$

where $G\left(\lambda_{j, n} t+\mu_{j, n}\right)=\left[G_{1}\left(\lambda_{j, n} t+\mu_{j, n}\right), G_{2}\left(\lambda_{j, n} t+\mu_{j, n}\right), \ldots\right.$, $\left.G_{N}\left(\lambda_{j, n} t+\mu_{j, n}\right)\right]$.

Also the initial condition will produce $m$ other equations:

$$
\sum_{n=0}^{m-1} a_{n, i} G(0)\left(P^{T}\right)^{i} C=d_{i}, \quad i=0,1, \ldots, m-1 .
$$

To find the solution $y_{N}(t)$ we collocate (47) at the collocation points $t_{j}=j /(N-m), j=1,2, \ldots, N-m$, to obtain

$$
\begin{aligned}
& G\left(t_{j}\right)\left(P^{T}\right)^{\alpha} C \\
& =\sum_{j=0}^{J} \sum_{n=0}^{m-1} p_{j, n}\left(t_{j}\right) G\left(\lambda_{j, n} t_{j}+\mu_{j, n}\right)\left(P^{T}\right)^{\beta_{n}} C \\
& \quad+g\left(t_{j}\right)
\end{aligned}
$$

for $j=1,2, \ldots, N-m$. Additionally, one can also use both the operational matrix of fractional derivative and delay operational matrix to solve problem (1). According to (44), we can approximate the delay function $y\left(\lambda_{j, n} t_{j}+\mu_{j, n}\right)$ and its fractional derivative using the operational matrices $\mathbf{P}$ and $\mathbf{R}$ as follows:

$$
\begin{aligned}
y_{N}\left(\lambda_{j, n} t_{j}+\mu_{j, n}\right) & =\mathbf{R} C^{T} G(t), \\
D^{\beta_{n}} y_{N}\left(\lambda_{j, n} t_{j}+\mu_{j, n}\right) & =\mathbf{R} C^{T} P^{\beta_{n}} G(t) .
\end{aligned}
$$

Putting this approximation together with (44) in (1), we have

$$
G(t)\left(P^{T}\right)^{\alpha} C=\sum_{j=0}^{J} \sum_{n=0}^{m-1} p_{j, n}(t) \mathbf{R} C^{T} P^{\beta_{n}} G(t)+g(t) .
$$


TABLE 2: Comparison errors obtained by the present method and those obtained in [25] when $\alpha=0.6$ and $\tau=0.3$ for Example 1 .

\begin{tabular}{lccc}
\hline$t$ & Error (new method) [25] & Error (FAM) [25] & Error (present method) \\
\hline 0.2 & 0.0781197 & 0.078155 & $3.37154 E-03$ \\
0.4 & 0.129928 & 0.129978 & $6.52102 E-03$ \\
0.6 & 0.190687 & 0.19076 & $9.77309 E-03$ \\
0.8 & 0.248601 & 0.248694 & $1.30349 E-02$ \\
1.0 & 0.307649 & 0.307763 & $1.64103 E-02$ \\
\hline
\end{tabular}

Thus, collocating (51) at the same collocation point as that in (47), we get

$$
\begin{aligned}
G\left(t_{j}\right)\left(P^{T}\right)^{\alpha} C= & \sum_{j=0}^{J} \sum_{n=0}^{m-1} p_{j, n}\left(t_{j}\right) \mathbf{R} C^{T} P^{\beta_{n}} G\left(t_{j}\right) \\
& +g\left(t_{j}\right) .
\end{aligned}
$$

Hence, (49) or (52) is $N-m$ nonlinear algebraic equation. Any of these equations together with (48) makes $N$ algebraic equations which can be solved using Newton's iterative method. Consequently, $y_{N}(x)$ given in (44) can be calculated.

\section{Numerical Examples}

In this section, some numerical examples are given to illustrate the applicability and accuracy of the proposed method. All the numerical computations have been done using Maple 18 .

Example 1. Consider the following example solved in [25]:

$$
\begin{aligned}
D^{\alpha} y(t)= & \frac{2}{\Gamma(3-\alpha)} y^{1-\alpha / 2}(t)+y(t-\tau)-y(t) \\
& +2 \tau \sqrt{y(t)}-\tau^{2}
\end{aligned}
$$

subject to

$$
y(t)=0, \quad t \leq 0 \text {. }
$$

The exact solution for this example is given by $y(t)=$ $t^{2}$. We solve the example when $\alpha=0.6$ and $\tau=0.3$. In Table 2, we compare the errors obtained by our method with those obtained using FAM and new approach in [25]. As reported in [25], the time required for the new method is 104.343750 seconds and for the FAM the time taken is 215.031250 seconds for completing the same task, whereas in our method we only need 38.080 seconds to complete the computations.

Example 2 (see [1]). Consider the following generalized fractional pantograph equation:

$$
D^{5 / 2} y(t)=-y(t)-y(t-0.5)+g(t), \quad t \in[0,1]
$$

subject to

$$
\begin{array}{r}
y(0)=0, \\
y^{\prime}(0)=0, \\
y^{\prime \prime}(0)=0,
\end{array}
$$

where

$$
g(t)=\frac{\Gamma(4)}{\Gamma(3 / 2)} t^{1 / 2}+t^{3}+(t-0.5)^{3} .
$$

The exact solution of this problem is known to be $y(t)=$ $t^{3}$. This problem is solved in [1] using generalized LaguerreGauss collocation scheme. We apply our technique with $N=$ 4. Approximating (55) with Genocchi polynomials, we have

$$
G(t)\left(P^{T}\right)^{5 / 2} C=-G(t) C+G(t-0.5) C+g(t) .
$$

Also from the initial conditions we have

$$
\begin{aligned}
G(0) C & =0, \\
G(0)\left(P^{T}\right) C & =0, \\
G(0)\left(P^{T}\right)^{2} C & =0 .
\end{aligned}
$$

Thus, collocating (58) at $t=0.267339$, we get

$$
\begin{aligned}
& -3.507078326+15.27479180 c_{4}+2 c_{1} \\
& +0.2727646328 c_{3}-1.930640400 c_{2}=0
\end{aligned}
$$

and (59) gives

$$
\begin{gathered}
c_{1}-c_{2}+c_{4}=0, \\
2 c_{1}-3 c_{2}=0, \\
6 c_{3}-12 c_{4}=0 .
\end{gathered}
$$

Solving these equations, we have

$$
\begin{aligned}
& c_{1}=0.4999969148, \\
& c_{2}=0.7499953722, \\
& c_{3}=0.4999969148, \\
& c_{4}=0.2499984574 .
\end{aligned}
$$

Thus, $y(t)=G(x) C$ is calculated and we have $0.9999938296 t^{3}$ which is almost the exact solution. In Table 3 , we compare the absolute errors obtained by our method (with only few terms $N=4$ ) and the absolute errors obtained in [1] when $N=22$ with different Laguerre parameters $\beta$. 
TABLE 3: Comparison of the absolute errors obtained by the present method and those obtained in [1] for Example 2.

\begin{tabular}{lcccc}
\hline$t$ & & $N=22[1]$ & & $N=4$ \\
& $\beta=2$ & $\beta=3$ & $6.273 E-06$ & $6.17040 E-09$ \\
\hline 0.1 & $1.030 E-04$ & $1.019 E-05$ & $3.892 E-05$ & $4.93630 E-08$ \\
0.2 & $6.510 E-04$ & $6.051 E-05$ & $1.023 E-04$ & $1.66600 E-07$ \\
0.3 & $1.740 E-03$ & $1.495 E-04$ & $1.901 E-04$ & $3.94910 E-07$ \\
0.4 & $3.283 E-03$ & $2.559 E-04$ & $2.944 E-04$ & $7.71300 E-07$ \\
0.5 & $5.138 E-03$ & $3.546 E-04$ & $4.088 E-04$ & $1.33280 E-06$ \\
0.6 & $7.175 E-03$ & $4.261 E-04$ & $5.306 E-04$ & $2.11640 E-06$ \\
0.7 & $9.303 E-03$ & $4.592 E-04$ & $6.597 E-04$ & $3.15920 E-06$ \\
0.8 & $1.147 E-02$ & $4.510 E-04$ & $7.977 E-04$ & $4.49820 E-06$ \\
0.9 & $1.367 E-02$ & $4.055 E-04$ & $9.468 E-04$ & $6.17040 E-06$ \\
1.0 & $1.589 E-02$ & $3.311 E-04$ & \\
\hline
\end{tabular}

TABLE 4: Comparison of the absolute errors obtained by the present method and those in [26] for Example 4.

\begin{tabular}{lcc}
\hline$t$ & Absolute error [26] $N=4$ & Absolute error (present method) $N=3$ \\
\hline 0.1 & $0.100 E-07$ & $1.15000 E-09$ \\
0.2 & $0.115 E-07$ & $2.00000 E-09$ \\
0.3 & $0.115 E-07$ & $2.55000 E-09$ \\
0.4 & $0.107 E-07$ & $2.80000 E-09$ \\
0.5 & $0.967 E-08$ & $2.70000 E-09$ \\
0.6 & $0.811 E-08$ & $2.40000 E-09$ \\
0.7 & $0.641 E-08$ & $1.70000 E-09$ \\
0.8 & $0.440 E-08$ & $8.00000 E-10$ \\
0.9 & $0.223 E-08$ & $5.00000 E-10$ \\
1.0 & $0.372 E-09$ & $2.00000 E-09$ \\
\hline
\end{tabular}

Example 3. Consider the following fractional pantograph equation:

$$
D^{1 / 2} y(t)=2 y\left(\frac{3 t}{2}\right)+\frac{8 t^{3 / 2}}{3 \sqrt{\pi}}-\frac{9 t^{2}}{2}, \quad t \in[0,1]
$$

subject to

$$
\begin{aligned}
& y(0)=0, \\
& y(1)=1 .
\end{aligned}
$$

The exact solution of this problem is known to be $y(t)=$ $t^{2}$. We solve (63) using our technique with $N=3$ only. As in Example 2, we obtained the values of the coefficients to be

$$
\begin{aligned}
& c_{1}=\frac{1}{2}, \\
& c_{2}=\frac{1}{2}, \\
& c_{3}=\frac{1}{3} .
\end{aligned}
$$

Thus, $y_{N}(t)=G(t) C$ is calculated to be $t^{2}$ which is the exact solution and so there is nothing to compare for the error is zero.
Example 4. Consider the following fractional pantograph equation solved in [26]:

$$
\begin{array}{rl}
D^{2} & y(t)+D^{3 / 2} y(t)+y(t) \\
& =y\left(\frac{t}{2}\right)+\frac{3 t^{2}}{4}+4 \sqrt{\frac{t}{\pi}}+2, \quad t \in[0,1]
\end{array}
$$

subject to

$$
\begin{aligned}
& y(0)=0, \\
& y(1)=1 .
\end{aligned}
$$

The exact solution of this problem is known to be $y(t)=$ $t^{2}$. As in Example 3, we solve (66) using our technique with $N=3$ and the values of the coefficients obtained are

$$
\begin{aligned}
& c_{1}=0.5000000011, \\
& c_{2}=0.5000000011, \\
& c_{3}=0.3333333384 .
\end{aligned}
$$

Thus, $y_{N}(t)=G(t) C$ is calculated and compared with the exact solution. This problem is solved using Taylor collocation method in [26] when $N=4,5$, and 6. In Table 4, we compare the absolute errors obtained by present method when $N=3$ with the errors obtained when $N=4$ in [26]. 


\section{Conclusion}

In this paper, a collocation method based on the Genocchi delay operational matrix and the operational matrix of fractional derivative for solving generalized fractional pantograph equations is presented. The comparison of the results shows that the present method is an excellent mathematical tool for finding the numerical solutions delay equation. The advantage of the method over others is that only few terms are needed and every operational matrix involves more numbers of zeroes; as such the method has less computational complexity and provides the solution at high accuracy.

\section{Conflicts of Interest}

The authors declare that there are no conflicts of interest regarding the publication of this manuscript.

\section{Acknowledgments}

This work was supported in part by MOE-UTHM FRGS Grant Vot 1433. The authors also acknowledge financial support from UTHM through GIPS U060. The third author would like to acknowledge Faculty of Computer Science and Information Technology, UNIMAS, for providing continuous support.

\section{References}

[1] A. H. Bhrawy, A. A. Al-Zahrani, Y. A. Alhamed, and D. Baleanu, "A new generalized laguerre-gauss collocation scheme for numerical solution of generalized fractional pantograph equations," Romanian Journal of Physics, vol. 59, no. 7-8, pp. 646-657, 2014.

[2] J. R. Ockendon and A. B. Tayler, "The dynamics of a current collection system for an electric locomotive," Proceedings of the Royal Society of London A: Mathematical, Physical and Engineering Sciences, vol. 322, pp. 447-468, 1971.

[3] W. G. Aiello, H. I. Freedman, and J. Wu, "Analysis of a model representing stage-structured population growth with statedependent time delay," SIAM Journal on Applied Mathematics, vol. 52, no. 3, pp. 855-869, 1992.

[4] M. Dehghan and F. Shakeri, "The use of the decomposition procedure of Adomian for solving a delay differential equation arising in electrodynamics," Physica Scripta, vol. 78, no. 6, article 065004, 2008.

[5] Y. Kuang, Delay Differential Equations with Applications in Population Dynamics, Academic Press, New York, NY, USA, 1993.

[6] E. Tohidi, A. H. Bhrawy, and K. Erfani, "A collocation method based on Bernoulli operational matrix for numerical solution of generalized pantograph equation," Applied Mathematical Modelling. Simulation and Computation for Engineering and Environmental Systems, vol. 37, no. 6, pp. 4283-4294, 2013.

[7] E. Yusufoglu, "An efficient algorithm for solving generalized pantograph equations with linear functional argument," Applied Mathematics and Computation, vol. 217, no. 7, pp. 3591-3595, 2010.

[8] Y. Yang and Y. Huang, "Spectral-collocation methods for fractional pantograph delay-integrodifferential equations,"
Advances in Mathematical Physics, vol. 2013, Article ID 821327, 14 pages, 2013.

[9] S. Yüzbasi and M. Sezer, "An exponential approximation for solutions of generalized pantograph-delay differential equations," Applied Mathematical Modelling. Simulation and Computation for Engineering and Environmental Systems, vol. 37, no. 22, pp. 9160-9173, 2013.

[10] S. Sedaghat, Y. Ordokhani, and M. Dehghan, "Numerical solution of the delay differential equations of pantograph type via Chebyshev polynomials," Communications in Nonlinear Science and Numerical Simulation, vol. 17, no. 12, pp. 4815-4830, 2012.

[11] S. Yüzbasi, N. Sahin, and M. Sezer, "A Bessel collocation method for numerical solution of generalized pantograph equations," Numerical Methods for Partial Differential Equations, vol. 28, no. 4, pp. 1105-1123, 2012.

[12] A. Isah and C. Phang, "Operational matrix based on Genocchi polynomials for solution of delay differential equations," Ain Shams Engineering Journal, 2017.

[13] C. Phang, N. F. Ismail, A. Isah, and J. R. Loh, "A new efficient numerical scheme for solving fractional optimal control problems via a Genocchi operational matrix of integration," Journal of Vibration and Control, 2017.

[14] S. Yüzbasi, "A collocation method for numerical solutions of fractional-order logistic population model," International Journal of Biomathematics, vol. 9, no. 2, article 1650031, 14 pages, 2016.

[15] S. Yüzbasi, "Numerical solutions of fractional Riccati type differential equations by means of the Bernstein polynomials," Applied Mathematics and Computation, vol. 219, no. 11, pp. 6328-6343, 2013.

[16] A. A. Kilbas, H. M. Srivastava, and J. J. Trujillo, "Preface," NorthHolland Mathematics Studies, vol. 204, pp. 7-10, 2006.

[17] I. Podlubny, Fractional Differential Equations: An Introduction to Fractional Derivatives, Fractional Differential Equations, to Methods of Their Solution and Some of Their Applications, vol. 198, Academic Press, San Diego, Calif, USA, 1999.

[18] S. Araci, "Novel identities for $q$-Genocchi numbers and polynomials," Journal of Function Spaces and Applications, vol. 2012, Article ID 214961, 13 pages, 2012.

[19] S. Araci, "Novel identities involving Genocchi numbers and polynomials arising from applications of umbral calculus," Applied Mathematics and Computation, vol. 233, pp. 599-607, 2014.

[20] T. Kim, "Some identities for the Bernoulli, the Euler and the Genocchi numbers and polynomials," Advanced Studies in Contemporary Mathematics, vol. 20, no. 1, pp. 23-28, 2010.

[21] A. Isah and C. Phang, "Genocchi Wavelet-like operational matrix and its application for solving non-linear fractional differential equations," Open Physics, vol. 14, no. 1, pp. 463-472, 2016.

[22] E. Kreyszig, Introductory Functional Analysis with Applications, vol. 81, John Wiley \& Sons, New York, NY, USA, 1989.

[23] A. Saadatmandi and M. Dehghan, "A new operational matrix for solving fractional-order differential equations," Computers \& Mathematics with Applications, vol. 59, no. 3, pp. 1326-1336, 2010.

[24] P. Chang and A. Isah, "Legendre Wavelet Operational Matrix of fractional Derivative through wavelet-polynomial transformation and its Applications in Solving Fractional Order Brusselator system," Journal of Physics: Conference Series, vol. 693, no. 1, article 012001, 2016. 
[25] V. Daftardar-Gejji, Y. Sukale, and S. Bhalekar, "Solving fractional delay differential equations: a new approach," Fractional Calculus and Applied Analysis, vol. 18, no. 2, pp. 400-418, 2015.

[26] A. Anapali, Y. Öztürk, and M. Gülsu, "Numerical Approach for Solving Fractional Pantograph Equation," International Journal of Computer Applications, vol. 113, no. 9, pp. 45-52, 2015. 


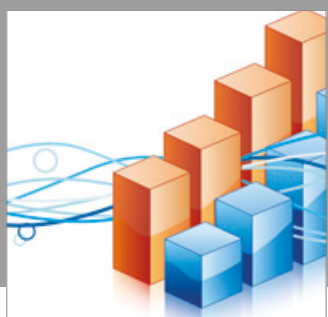

Advances in

Operations Research

vatersals

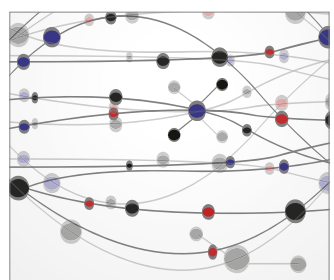

\section{The Scientific} World Journal
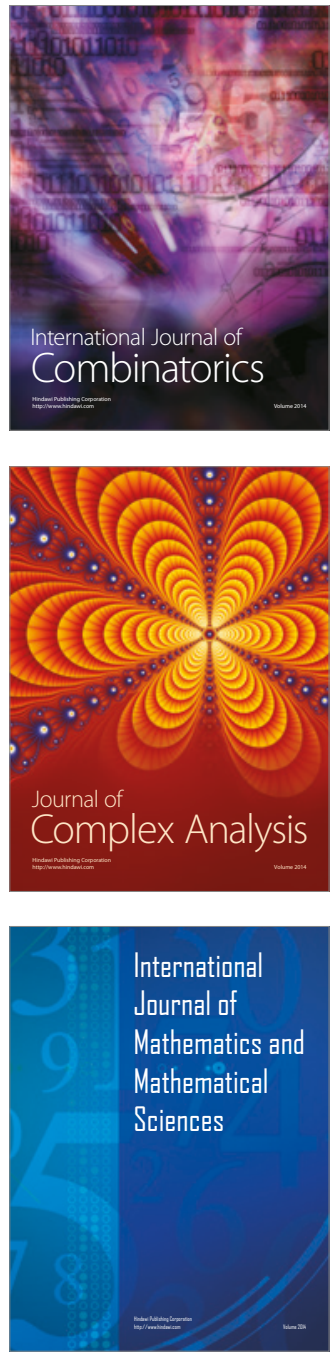
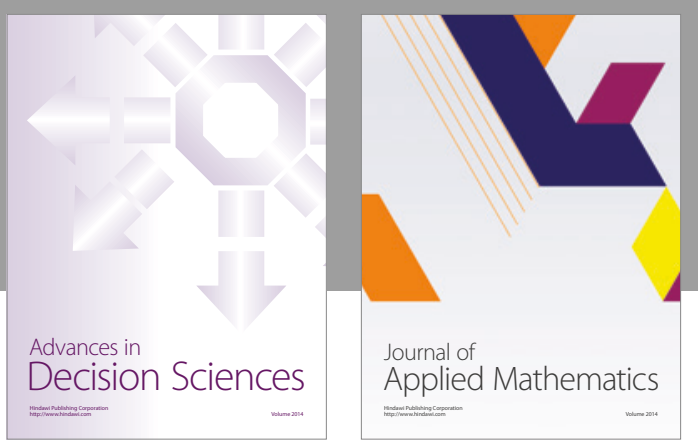

Algebra

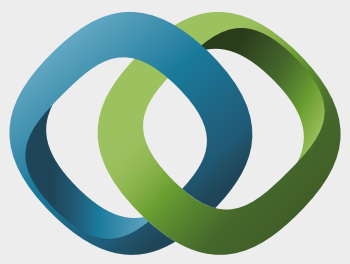

\section{Hindawi}

Submit your manuscripts at

https://www.hindawi.com
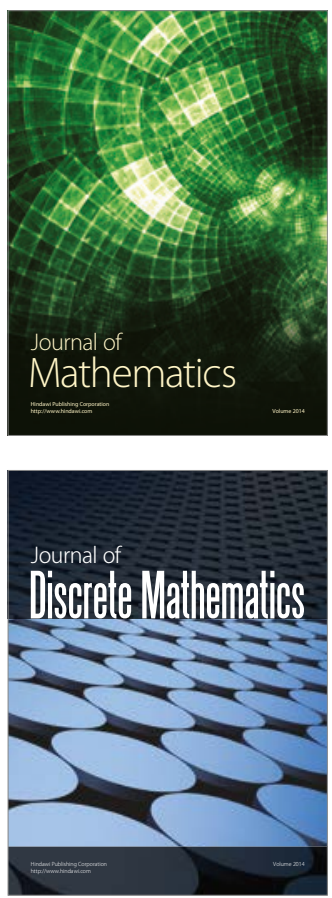

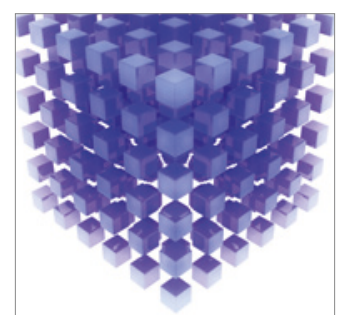

Mathematical Problems in Engineering
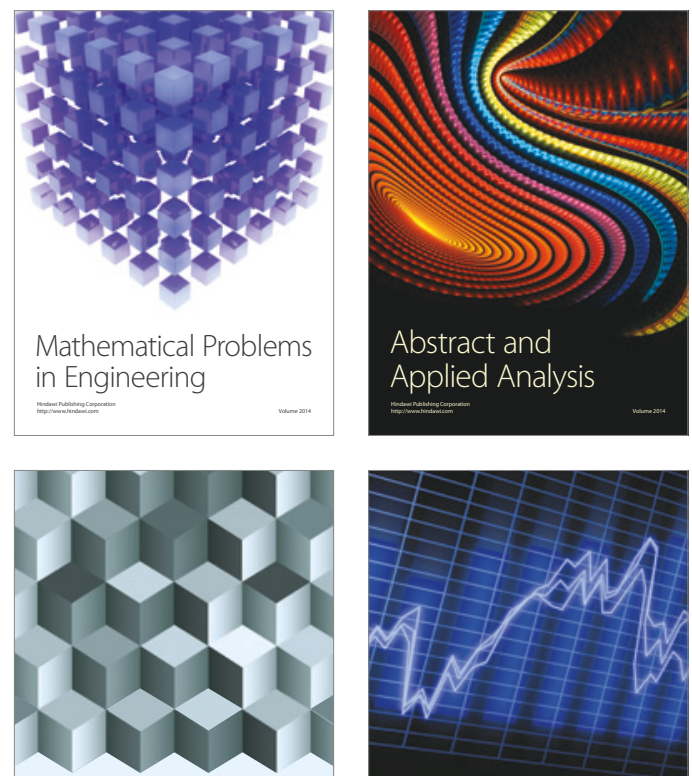

Journal of

Function Spaces

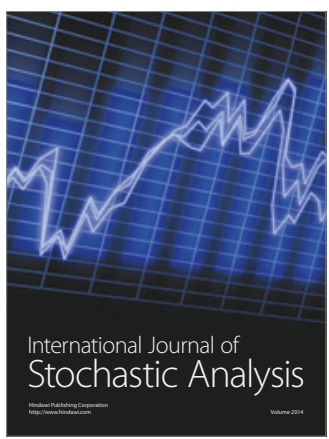

Probability and Statistics
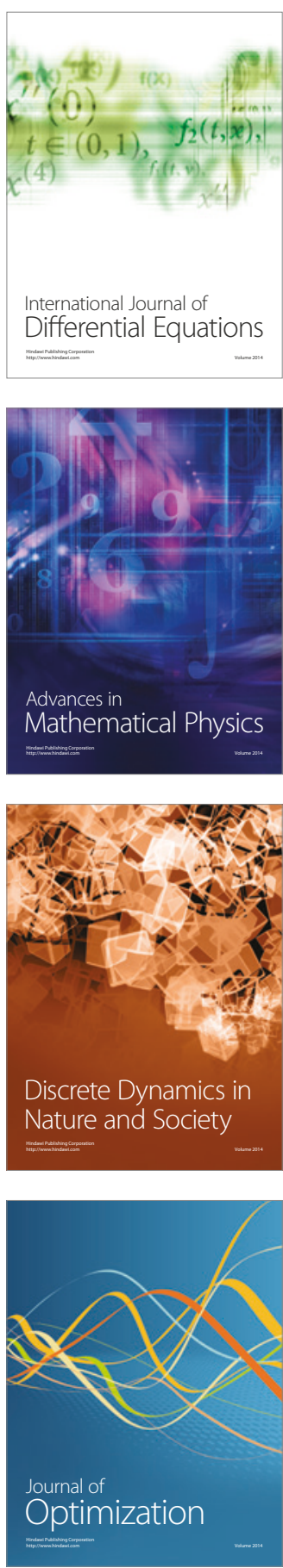